\title{
«Un salto de Nijinski no puede ser contado » Ortega y Gasset, spectateur des Ballets russes
}

La recepción de los Ballets russes en Madrid y Barcelona (1916-1929)

The reception of the Ballets Russes in Madrid and Barcelona (1916-1929)

\section{Hélène Frison}

\section{OpenEdition}

\section{Journals}

Édition électronique

URL : http://journals.openedition.org/bhce/610

DOI : $10.4000 /$ bhce. 610

ISSN : 1968-3723

\section{Éditeur}

Presses Universitaires de Provence

\section{Édition imprimée}

Date de publication : 1 décembre 2016

Pagination : 221-233

ISSN : 0987-4135

\section{Référence électronique}

Hélène Frison, « « Un salto de Nijinski no puede ser contado » Ortega y Gasset, spectateur des Ballets russes », Bulletin d'Histoire Contemporaine de l'Espagne [En ligne], 50 | 2016, mis en ligne le 09 octobre 2018, consulté le 18 septembre 2020. URL : http://journals.openedition.org/bhce/610 ; DOI : https:// doi.org/10.4000/bhce.610 


\title{
"Un salto de Nijinski no puede ser contado " Ortega y Gasset, spectateur des Ballets russes
}

\author{
Hélène FRISON
}

Université Paris 3 - Sorbonne-Nouvelle

$\mathrm{D}$ ans sa volonté de tout connaître et de tout confronter, Ortega y Gasset ne pouvait ignorer la rénovation de la scène, et plus généralement des arts, qui s'opérait avec les Ballets russes. Et bien qu'il avouât ne rien connaître à la musique', il y consacra trois articles publiés dans El Sol, en 1921. Deux d'entre eux sont directement liés à l'écriture de son célèbre essai, La Deshumanización del arte, essai initialement publié par chapitre dans le même journal en 1924, avant d'être repris d'un seul tenant l'année suivante dans La Revista de Occidente.

Auteur prolifique s'il en est, Ortega y Gasset s'est vu reprocher son manque de systématicité et l'hétérogénéité de ses écrits. De fait, il n'existe aucun ouvrage dans lequel sa pensée soit exposée de manière exhaustive. Malgré cette apparente "dissémination » de la pensée ortéguienne, Julián Marías² d'abord, puis Charles Cascalès ensuite, affirment néanmoins que l'ensemble de ses articles, essais et conférences, est vertébré par le concept de la raison vitale:

Son intérêt ne s'est pas limité à des questions strictement philosophiques et tous les thèmes ont attiré son attention, qu'il s'agisse d'art, de politique ou d'histoire. Mais tous ses écrits se trouvent subordonnés à une intention philosophique déterminée qui en est le lien vivant et c'est seulement à la lumière de cet axe général et fondamental qu'ils deviennent pleinement intelligibles. En dépit de leur caractère disparate, les œuvres du penseur espagnol contiennent, sans équivoque possible, les lignes directrices d'un véritable système philosophique dont toutes les parties rayonnent autour d'une intuition centrale ${ }^{3}$.

L' « intuition philosophique » dont parle Cascalès peut se résumer ainsi : redonner au moi son individualité vécue sans l'isoler des valeurs rationnelles. Ortega y Gasset entend dépasser la traditionnelle antinomie qui oppose idéalisme et réalisme et affirme le principe de non-contradiction entre la vie et la raison. Le philosophe pose comme point de départ la totalité suivante: « un homme dans ses circonstances réfléchissant sur le monde »" Ce rapport entre un homme concret et défini, et un monde également concret et défini, est le cœur de la pensée ortéguienne qui se trouve synthétisée dans le célèbre aphorisme « je suis moi et ma circonstance ». À cette totalité, Ortega y Gasset apporte la réponse de la raison vitale. Seule l'union dynamique de la vie et de la raison permet de dépasser l'opposition entre la spontanéité et le rationnel, entre la multiplicité du donné et le caractère unique de la vérité.

1 «Yo no entiendo nada de música », José Ortega y Gasset « Musicalia II », El Sol, 24 mars 1921, p. 3.

2 Julián Marias, Introducción a la filosofía, Madrid, Revista de Occidente, 1947.

3 Charles Cascalés, L'humanisme d'Ortega y Gasset, Paris, Presses Universitaires de France, 1957, p. 5.

4 Jean-Paul Borel, Raison et vie chez Ortegay Gasset, Neuchâtel, Éditions de la Baconnière, 1959, p. 18. 
L'explicitation de la célèbre formule - « je suis moi et ma circonstance » - a abouti à l'engagement volontaire et réfléchi du philosophe dans la réalité espagnole. Les domaines politiques, culturels, littéraires, scientifiques, etc. qu'analyse Ortega sont autant d'éléments concrets qui se posent à lui et auxquels il se confronte au moyen de la raison. Convaincu que la multiplicité des perspectives est la seule possibilité d'approcher la véritable « raison absolue ", Ortega choisit de n'ignorer aucun aspect de la réalité. Il est tout à la fois journaliste, professeur, politicien, homme du monde, etc. Et afin d'assurer un contact plus étroit avec ses compatriotes, il choisit de s'exprimer principalement dans les journaux.

Au sein des nombreux domaines qu'Ortega embrasse, la culture occupe une place toute particulière. Opposée à la nature de la vie, celle-ci constitue une tentative d'interprétation que l'homme donne à son existence; elle est l'effort qu'il réalise pour résoudre sa propre énigme. Ce qui explique son caractère originellement authentique: «Elle jaillit du cœur même de l'homme, geste défensif contre l'univers où il est englouti " ${ }^{5}$. Contrairement à la tradition qui s'hérite et se transmet, l'établissement de la culture devient rapidement synonyme de sclérose pour la génération qui ne l'a pas créée. À un moment donné, et puisque différentes générations coïncident à cet instant précis, une culture sclérosée et une culture jaillissante coexistent.

Écrits en 1921, les cinq articles concernant les Ballets russes s'inscrivent pleinement au sein du système ortéguien et illustrent, à leur mesure, la volonté du philosophe de saisir la « crise culturelle que traverse l'Occident $»^{6}$.

Dans « Apatía artística ${ }^{7}$ et « Elogio del Murciélago I y II ${ }^{8}$, l'auteur met en évidence cette coexistence de deux cultures en Espagne. L'une d'elle a été héritée des générations antérieures et ne correspond plus à la sensibilité actuelle. L'autre, celle qui témoigne d'un changement d'attitude radicale par rapport à la vie, est à mettre au crédit - entre autres - des Ballets russes. Dans les deux articles réunis sous le titre « Musicalia » ${ }^{9}$, Ortega y Gasset analyse les raisons de l'impopularité de la musique nouvelle, et plus précisément celle de Debussy et celle de Stravinsky.

La critique actuelle a fréquemment analysé conjointement les articles suivants ${ }^{10}$ : «Musicalia I y II » et "Apatía artística» et ce, pour deux raisons. L'une tient à la proximité des dates de publication (1921), la seconde au fait qu'ils traitent tous trois d'une thématique fort peu étudiée par le philosophe, celle de la musique. Nous choisissons, pour notre part, de procéder différemment en étudiant dans un premier temps, "Apatía artística » auquel nous joignons « Elogio del Murciélago I y II ». Ce premier groupement fonctionne en miroir puisque l'auteur oppose à « l'apathie » provoquée par les spectacles traditionnels espagnols, le plaisir esthétique que suscite la scène russe. D'un simple point

5 Charles Cascalès, Id., p. 136.

6 Paul AUBERT, préface à La déshumanisation de l'art, suivi de Idées sur le roman, et de L'art au présent et au passé de José OrTEGa y Gasset (trad., étude préliminaire et notes de Paul Aubert et Ève Giustiniani, Cabris, Éd. Sulliver, 2008, p. 7.

7 José Ortega y Gasset, « Apatía artística », El Sol, 18 octobre 1921, p. 3.

8 José Ortega y Gasset, « Elogio del Muerciélago I », El Sol, 6 novembre 1921, p. 3 ; « Elogio del Murciélago II », El Sol, 18 novembre 1921, p. 3.

9 José Ortega y Gasset, « Musicalia I », El Sol, 8 mars 1921, p. 3 ; « Musicalia II », El Sol, 24 mars 1921, p. 3 .

10 José M. Garcia LABORDA, « Los escritos musicales de Ortega y Gasset y su « circunstancia » histórica », Revista de Estudios Orteguianos, n¹0-11, mai et noviembre 2005, p. 245-272; Clementina CantIllo, "Vida, cultura, arte : la música en le pensamiento de Ortega y Gasset », Revista de Estudios Orteguianos, $n^{\circ} 23$, noviembre 2011, p. 107-124. 
de vue chronologique, ces trois articles sont publiés sur une période d'un mois. Dans un second temps, nous analyserons «Musicalia I y II », puisque ce corpus s'intéresse plus précisément à la musique nouvelle et à son caractère minoritaire, thématique qui sera reprise et développée par la suite dans La deshumanización del arte.

\section{Culture sclérosée - vs - culture jaillissante}

Publié le 18 octobre 1921, "Apatía artística» se propose d'analyser les causes de l'absence d'émotion que provoquent désormais les concerts et les expositions présentés en Espagne. Selon Ortega y Gasset, la musique et la peinture représentées, malgré des qualités intellectuellement objectives, ne suscitent plus aucune émotion esthétique car elles ne sont plus en accord avec la sensibilité de leur public:

On pourrait dire que, soudainement, l'ensemble de la musique - ancienne et nouvelle

- et que toute la peinture se sont vitalement disloqués de nous et sont devenus des faits indifférents qui se réalisent hors de notre sphère d'affectivité11.

Deux raisons essentielles expliquent cette " vacuité émotionnelle ». Premièrement, cet art sclérosé ne plonge plus ses racines dans l'intimité du spectateur et se voit donc coupé de son élan vital. Il s'impose malgré lui à celui qui l'écoute ou le regarde. Deuxièmement, ce sentiment d' «apathie » est commun à tous les pays occidentaux. Il témoigne donc d'un changement collectif de sensibilité et non d'une dégénérescence propre à une société précise.

Or, selon Ortega y Gasset toute culture marquant un écart infranchissable entre la sincérité de son public et ses idéaux doit être rejetée. Prendre conscience du décrochage qui existe entre ce qui est (re) présenté et l'apathie ressentie consiste ni plus ni moins à faire acte de loyauté. Mais pour Ortega y Gasset cette exigence ne peut être assumée que par une minorité:

Je crois que la majorité des hommes vit une existence intérieure, d'une certaine manière, apocryphe. Leurs opinions, en réalité, ne sont pas leurs propres opinions, mais des états de convictions dont ils sont contaminés par l'extérieur, et ce qu'ils croient ressentir n'est pas réellement ressenti; il s'agit plutôt d'émotions étrangères qui se répercutent en eux. Seules quelques individualités d'une condition supérieure possèdent ce talent particulier de distinguer intérieurement ce qui est authentique de ce qui est apocryphe ${ }^{12}$.

L'assimilation du progrès, des découvertes scientifiques et des bouleversements que traverse l'Europe au lendemain de la Première Guerre transforme le rapport qui liait l'homme au monde et par là même, ébranle l'art et la pensée, comme le précise Paul Aubert13. Les formes artistiques héritées du passé sont dorénavant caduques, incapables de dessiner le monde tel qu'il est. Les artistes se retrouvent ainsi dans l'obligation d'inventer

11 «Diríase que, de pronto, la música toda-vieja y mueva-, que toda la pintura han quedado desarticuladas vitalmente de nosotros y se han convertido en hechos indiferentes que acontecen fuera de muestra esfera de afectividad », José OrTeGa y GASSET, « Apatía artística », El Sol, 18 octobre 1921, p. 3).

12 «Yo creo que la mayor parte de los hombres vive una vida interior, en cierta manera, apócrifa. Sus opiniones no son, en verdad, sus opiniones, sino estados de convicción que reciben de fuera por contagio, y lo que creen sentir no lo sienten realmente, sino que, más bien, dejan repercutir en su interior emociones ajenas. Sólo ciertas individualidades de selecta condición poseen el peculiar talento de distinguir dentro de sí lo auténtico de los apócrifo » (José ORTEGA Y GASSET, "Apatía artística », El Sol, 18 octobre 1921, p. 3.

13 Paul Aubert, étude préliminaire à La déshumanisation de l'art, op. cit., p. 10. 
de nouveaux modèles afin de pouvoir exprimer les réalités inédites auxquelles l'homme se trouve confronté.

Dans le domaine musical, Ortega juge que ce changement de sensibilité artistique se caractérise par une restriction des attentes et des formes: "Le diaphragme de notre ressenti artistique s'est contracté et les émotions qu'il laisse entrer sont non seulement moins nombreuses, mais également moins volumineuses qu'avant $»^{14}$. Citant l'exemple de Wagner, Ortega y Gasset revient sur la conception transcendantale de l'art qui parcourt le $\mathrm{XIX}^{\mathrm{e}}$ siècle. L'art, et tout particulièrement la musique, s'est coupé de la vie et est devenu une valeur indépendante qui jouit d'une autorité spécifique. Schopenhauer, par exemple, présente la musique comme une "langue éminemment universelle [...] [qui] exprime d'une seule manière, par les sons, avec vérité et précision, l'être, l'essence du monde ${ }^{15}$. Elle permet en un mot d'aller « au-delà des Idées »" ${ }^{16}$, ce que récuse Ortega y Gasset avec véhémence:

N'est-ce pas une aberration évidente que d'attendre autant des sons combinés? Un chef d'orchestre peut-il diriger le cœur humain, la société et l'histoire? Une mélodie peutelle se substituer à une religion? La monstruosité de cette exagération musicale osée par Wagner reste consignée à ce siècle de la démesure systématique qu'est le XIX ${ }^{c}$. Époque de l'impérialisme généralisé, il n'y a pas eu une partie d'elle-même qui n'ait voulu régner sur les autres, être la première ou la seule ${ }^{17}$.

Utilisant l'image d'une organisation hiérarchique dans "Apatía artística », puis d'un système de cercles concentriques dans La deshumanización del arte, « dont le rayon mesure la distance dynamique à l'axe de notre vie $»^{18}$, Ortega explique comment le $\mathrm{XIX}^{\mathrm{c}}$ siècle constitue une exception au sein de l'ordonnance des activités humaines. Après avoir occupé pendant un siècle une place proche du centre cordial ${ }^{19}$, la musique, dans l'époque actuelle, tend à s'éloigner vers la périphérie pour occuper une place secondaire, qui lui correspond par nature. Soumis à la tyrannie de la transcendance, l'art aurait donc occupé

14 « El diafragma de nuestro sentir artístico se ha hecho más angosto, y las emociones a que deja paso no sólo son menos numerosas que antaño, sino también de menor calibre 》 (José ORTEGA Y GASSET, " Apatía artística », op. cit., p. 3).

15 Arthur SchopenhaUER, Le monde comme volonté et comme représentation [1819], livre III, § 52, (trad. Auguste Burdeau), Paris, Presses universitaires de France, 1966, p. 337 - 338.

16 Arthur Schopenhauer, Id., p. 329.

17 « ¿No es a todas luces monstruoso esperar tanto de los sonidos concertados? ¿Puede un director de orquesta dirigir el corazón humano, la sociedad y la historia? ¿Puede una melodía sustituir a una religión? Quedaba consignada al siglo XIX, centuria del desmesuramiento en todo, la monstruosidad de este superlativo musical osado por Wagner. Edad de imperialismo omnímodo, no hubo en ella cosa que no quisiera imperar a las demás, ser la primera o la única. » (José OrTEGA Y GASSET, « Apatia artistica ", op. cit., p. 3).

18 « Pueden representarse como una serie de círculos concéntricos, cayo radio mide la distancia dinámica al eje de muestra vida, donde actúan muestros supremos afanes. " (José ORTEGA Y GASSET, " La deshumanización del arte», Obras completas, t. III (1917-1925), 2012 [2005], Madrid, Taurus, p. 876 ; trad. Paul Aubert et Ėve Giustiniani, La déshumanisation de l'art, op. cit., p. 109).

19 Cette supériorité de la musique sur les autres arts a également été développée par Schopenhauer, comme le souligne Ortega dans ce même article: "La musique, en effet, est une objectivité, une copie quasi immédiate de toute la volonté que l'est le monde, que le sont les Idées elles-mêmes [...]. Elle n'est donc pas, comme les autres arts, une reproduction des Idées, mais une reproduction de la volonté au même titre que les Idées elles-mêmes. C'est pourquoi l'influence de la musique est plus puissante et plus pénétrante que celle des autres arts; ceux-ci n'expriment que l'ombre, tandis qu'elle parle de l'être ", Arthur SCHOPENHAUER, Le monde comme volonté et comme représentation, op. cit., p. 329. 
une place indue tout au long du XIX ${ }^{\mathrm{e}}$ siècle, place qui lui aurait auparavant été interdite par la prééminence des activités plus proprement spirituelles:

L'art [avant le $\left.\mathrm{XIX}^{\mathrm{e}}\right]$, sous toutes ses formes, était perçu comme appartenant à une sphère inférieure à celle de la religion et de la pensée. Au sein de la sphère artistique, la musique et la peinture se plaçaient loin derrière la poésie. Ce qui est important selon cette perspective, c'est que personne n'attendait de la musique et de la peinture qu'elles suscitent des émotions d'une qualité et d'un niveau correspondants aux activités de premier ordre. Elles constituaient simplement d'agréables passe-temps, de ravissants ingrédients du paysage vital ${ }^{20}$.

Dans «Apatía artística, », Ortega cite Stravinsky comme paradigme de la musique nouvelle sans pour autant préciser ce qui caractérise son art. Quelques jours plus tard, il consacre, cependant, deux articles aux spectacles russes - le théâtre de la Chauve-Souris et la compagnie de Diaghilev - qu'il présente comme l'exact contrepoint des spectacles traditionnels sclérosés.

À l'opposé des représentations traditionnelles - théâtre et opéra en tête -, les Ballets russes et la Chauve-Souris procurent un véritable plaisir esthétique aux spectateurs, grâce à la rethéâtralisation de la scène qu'ils opèrent. Ortega y Gasset confronte ici point par point les deux types de spectacles. Les spectacles traditionnels se limitent à «mettre en scène » le texte, à matérialiser ce que dit l'œuvre sans chercher à susciter l'imagination du public. Ils sont donc redondants et superflus à force de paraphrase. À l'inverse, les Ballets russes donnent la suprématie à l'œil et à l'oreille et produisent un spectacle qui ne peut se passer de la scène: «Un saut de Nijinski ne peut pas être raconté. Le passage de sa réalisation à sa description anéantit toute sa valeur $»^{21}$.

Outre leur nature originellement physique, ces spectacles nouveaux se différencient également par leur fort pouvoir de suggestion. Ici, point d'approche mimétique du réel comme il est de mise dans les spectacles traditionnels. La seule préoccupation est d'ordre esthétique. Il s'agit de créer l'illusion en fusionnant les genres et les moyens afin de créer une fantasmagorie bannissant toute figuration de l'homme. Ces nouveaux spectacles produisent un plaisir esthétique car ils sont évasion et non explicitation. Ils sollicitent l'intuition du spectateur et non plus sa raison:

Non seulement ils nous montrent quelque chose que nous ne pouvons voir qu'au théâtre, mais ils acceptent le caractère de l'illusion, de l'irréalité propre à l'art, et ils élèvent sur les misérables planches un édifice de songes, une faune et une flore exclusivement théâtrales et non réelles ${ }^{22}$.

Enfin, selon Ortega y Gasset, l'art permet de percevoir les changements de sensibilité collective. En tant qu'activité « libre », il est «moins étroitement soumis aux conditions

20 « El arte, en todas sus formas, era sentido como un orbe inferior al de la religión y al del pensamiento. Dentro del orbe artístico, música y pintura se alzaban a larga distancia detrás de la poesía. Lo importante de esta perspectiva es que nadie pedia a la música y a la pintura emociones de calidad $y$ valor correspondientes a las actividades de primer orden. Eran sólo deleitables pasatiempos, encantadores ingredientes del paisaje vital. » (José ORTEGA y GASSET, « Apatía artística », op. cit..).

21 «Un salto de Nijinsky no puede ser contado. Al pasar de su ejecución a su descripción se volatiliza cuanto hay de valor », José OrTEgA y Gasset, "Elogio del Murciélago II », 18 novembre 1921, p. 3.

22 « No solamente nos presentan algo que sólo en el teatro podemos ver, sino que aceptan el carácter de farsa, de irrealidad propio al arte, y levantan sobre el misero tablado un edificio de ensoñaciones, una fauna y una flora exclusivamente teatrales y no reales » (José ORTEGA Y GASSET, « Elogio del Murciélago II ", op. cit..). 
sociales de chaque époque $»^{23}$. " Dis-moi comment tu te divertis et je te dirai qui tu es » annonce-t-il dans « Elogio del Murciélago $»^{24}$. Ainsi, la décadence des spectacles espagnols est-elle analysée à l'aune de l'état de décrépitude d'une société :

Que personne ne s'étonne que cette capacité à feindre l'adaptation à des formes qui ont perdu pour nous leur vertu et leur prestige se manifeste dans l'ensemble de la vie contemporaine. Si nous ne sommes pas sincères avec nos plaisirs, comment pourrionsnous l'être avec le reste? Nous nous affilions à des partis politiques dont les programmes n'éveillent plus notre enthousiasme, nous maintenons des institutions empaillées, nous lisons des auteurs classiques que nous ne comprenons pas ou qui nous parlent de ce qui ne nous intéresse pas, etc., etc. ${ }^{25}$.

L'inactualité des spectacles n'est pas un phénomène isolé. Il est symptomatique d'un changement d'attitude radical par rapport à la vie qui voit la notion classique de l'homme se disloquer. "Pour la première fois depuis le XvII ${ }^{\circ}$ siècle - explique Charles Cascalès il faut de nouveau refaire l'homme: l'art, la science, la politique et tout le reste doivent renaître ${ }^{26}$. En tant qu'expression spontanée, les spectacles russes révèlent l'écart sans cesse grandissant entre deux sensibilités et deux époques. Dans une Europe en mutation, ils apparaissent comme les précurseurs des temps à venir :

C'est que, tandis que l'Europe continue de pousser sans foi les momies de ses institutions et les spectres de ses fêtes exsangues, la Russie fait la révolution et danse. Là-bas, au fin fond de la planète, l'énorme corps slave se contorsionne, et nous l'imaginons comme un reptile d'avant le déluge qui tord la cordillère de ses vertèbres. [...]

Le Comité des Délégués Ouvriers et des Soldats qui a initié la grande révolte se présente à nous, qu'on le veuille ou non, sous la forme d'un chœur de danseurs, avec ses bottes vernies, ses longs manteaux d'astrakan et la musique de Stravinsky tandis que, assistant à une représentation de Petrouchka la masse palpitante et rythmique du peuple qui envahit la scène nous semble être une vue de la révolution pétersbourgeoise prise depuis le faubourg ${ }^{27}$.

23 « En otro lugar he indicado que el arte y la ciencia pura, precisamente por ser la actividades más libres, menos estrechamente sometidas a las condiciones sociales de cada época, son los primeros hechos donde puede vislumbrarse cualquier cambio de la sensibilidad colectiva. " (JOsé ORTEGA Y GASSET, «La deshumanización del arte », op. cit., p. 870 , trad. Paul AuBERT et Ėve GiustinIANI, La déshumanisation de l'art, op. cit., p. 100).

24 "Dime cómo te diviertes y te diré quién eres », José Ortega y GaSSET, «Elogio del Murciélago I », op. cit.

25 « Nadie extrañe que esta capacidad de fingir la adaptación a formas que para nosotros mismos han perdido virtud y prestigio se manifieste en toda la vida contemporánea. Si somos insinceros en muestros placeres, ¿cómo no lo seremos en todo lo demás? Nos inscribimos en partidos políticos cuyos programas no despiertan ya nuestro entusiasmo, conservamos instituciones disecadas, leemos autores clásicos que no entendemos o que nos hablan de lo que no nos importa, etc., etc. 》 (José ORTEGA Y Gasset, « Elogio del Murciélago I », op. cit..).

26 Charles Cascalés, L'humanisme d'Ortega y Gasset, op. cit., p. 160.

27 « Ello es que, en tanto Europa sigue empujando sin fe las momias de sus instituciones y los espectros de sus fiestas exangües, Rusia revoluciona y danza. Allá, al fondo del planeta, el enorme cuerpo eslavo se contorsiona, y lo imaginamos como un saurio prediluvial que retuerce la cordillera de sus vértebras. [...] El Comité de Delgados Obreros y Soldados que inició la gran revuelta se nos presenta, queramos o no, bajo la especie de un coro de danzarines, con sus botas altas de charol, largos abrigos de astracán y música de Stravinsky, mientras que, asistiendo a la ejecución de Petruchka, la masa del pueblo palpitante y rítmico que imunda la escena nos parece una vista de la revolución petersburguesa tomada desde un arrabal. », (José Ortega y GASSET, « Elogio del Murciélago », op. cit..). 


\section{De l'impopularité de la musique nouvelle}

Moins de dix jours avant l'ouverture de la cinquième saison russe au Teatro Real, Ortega y Gasset consacre son premier article à la musique. Le 8 mars 1921, le philosophe publie un papier dans lequel il s'interroge sur les raisons de l'impopularité de la musique nouvelle en citant en exemple, Debussy et Stravinsky. Lorsque, trois ans plus tard, il rédige son essai La deshumanización del arte, Ortega se souvient de cette étude sociologique - et non esthétique - de la musique pour analyser les points communs qui unissent les avant-gardes, malgré leur diversité apparente:

La fécondité d'une sociologie de l'art me fut révélée inopinément lorsqu'il y a plusieurs années j'eus l'idée un jour d'écrire quelque chose sur la nouvelle époque musicale qui commence avec Debussy. Je me proposais de définir le plus clairement possible la différence de style entre la nouvelle musique et la traditionnelle. Le problème était rigoureusement esthétique, et cependant, je trouvais que le chemin le plus court jusqu'à celui-ci était celui qui partait d'un phénomène sociologique: l'impopularité de la nouvelle musique ${ }^{28}$.

Dans «Musicalia », Ortega revient sur les réactions que suscita la création d'Iberia de Debussy lors du concert donné le 24 janvier 1921, pour comprendre les caractéristiques de la musique nouvelle. Afin d'étayer son raisonnement, le philosophe établit un parallèle avec le rejet que provoqua la musique de Wagner à ses débuts. Pour lui, les deux phénomènes obéissent à une mécanique fort différente. Si la défiance initiale envers Wagner était à mettre sur le compte d'un temps d'adaptation à une forme jusqu'alors inconnue, il en va tout autrement pour la musique nouvelle qui exige un positionnement nouveau de son public.

La musique romantique tire ainsi son origine d'un référent extramusical, souvent autobiographique. La musique nouvelle, quant à elle, trouve son principe en elle-même. Elle est un art intrinsèquement immanent qui tire sa justification du seul plaisir esthétique qu'il suscite. L'expression de l'intime en est bannie; la présence du réel, honnie. La difficulté qu'elle pose tient en réalité à son hermétisme plus qu'à un quelconque enchevêtrement structurel. Car, paradoxalement, la musique nouvelle tend à la simplification: "Personne, en effet, ne laissera, je pense, de reconnaître que Beethoven et Wagner, tout à fait populaires, sont incomparablement plus compliqués que l'impopulaire auteur de Pelléas et Mélisande. "C'est simple comme bonjour », a dit Cocteau en parlant de la musique nouvelle $»^{29}$.

La musique nouvelle requiert une attitude distincte de la part de son auditeur. Pour la goûter, ce dernier doit se départir de son attitude habituelle, qui l'incite à rechercher du connu ou du réel. Il doit se tourner entièrement vers la contemplation esthétique:

28 «La fecundidad de una sociología del arte me fue revelada inesperadamente cuando hace unos años me ocurrió un día escribir algo sobre la nueva época musical, que empieza con Debussy. Yo me proponía definir con la mayor claridad posible la diferencia de estilo entre la muevo música y la tradicional. El problema era rigorosamente estético, $y$, sin embargo, me encontré con que el camino más corto hasta él partía de un fenómeno sociológico: la impopularidad de la mueva música. " (José ORTEGA y GASSET, «La deshumanización del arte », op. cit., p. 847 ; trad. Paul Aubert et Ève Giustiniani, La déshumanisation de l'art, op. cit., p. 66).

29 «Porque nadie, pienso, desconocerá que Beethoven y Wagner, populares, son incomparablemente más complicados que el impopular autor de Pelléas. "C'est simple comme bonjour» - ha dicho recientemente Cocteau hablando de la nueva música -. ", OrTEGA y Gasset « Musicalia I », op. cit. ; trad. Mathilde Pomés, José Ortega y Gasset. Essais Espagnols, Paris, Éditions du Cavalier, 1932, p. 130. 
Au lieu d'en écouter en nous l'écho sentimental [de la musique de Debussy et de Stravinsky, notre ouïe et notre attention tout entière se concentrent sur les sons euxmêmes, sur l'événement enchanteur qui se passe réellement à l'orchestre. Recueillant chaque sonorité, nous la dégustons, nous en apprécions la couleur et presque la forme ${ }^{30}$.

À l'inverse de la musique romantique qui suscite un sentiment d'empathie chez celui qui l'écoute, la musique nouvelle est donc exclusivement plaisir esthétique. La première provoque chez l'auditeur un mouvement vers l'intérieur, un repli sur soi qui permet de jouir de l'écho que trouvent en lui les sentiments évoqués par la composition ${ }^{31}$ :

La Romance en $\mathrm{fa}$ de Beethoven ou tout autre musique typiquement romantique, nous en jouissons concentrés vers le dedans. Tournant pour ainsi dire le dos à ce qui se passe dans le violon, nous n'avons d'attention que pour le flot d'émotions qu'il suscite en nous. Ce n'est pas la musique en elle-même qui nous intéresse, c'est sa répercussion mécanique en nous [...]. Nous ne jouissons pas en quelque sorte de la musique, mais de nous-mêmes. [...] Je dirai presque que nous entendons la Romance en $f a$, mais que nous écoutons notre chant intime $\mathrm{e}^{32}$.

La seconde, en revanche, implique une ouverture vers l'extérieur, vers la contemplation d'un élément étranger par son refus de prêter allégeance à une quelconque réalité connue. Le plaisir qu'elle suscite n'est donc pas immédiatement perceptible. L'auditeur doit fournir un effort de compréhension et conquérir le privilège d'y accéder:

Très simple dans ses procédés, elle [la musique nouvelle] s'inspire d'une attitude spirituelle radicalement opposée à celle du vulgaire. Si bien qu'elle n'est pas impopulaire parce que difficile; elle est difficile parce qu'impopulaire ${ }^{33}$.

Cette conception selon laquelle la beauté réside dans la musique même et non dans les sentiments qu'elle éveille en nous n'est pas nouvelle. Hanslick dans son essai Du beau dans la musique $(1854)^{34}$, avait posé les prémices du concept de la musique pure. La différence radicale qui existe entre ces deux conceptions réside dans la lecture sociologique réalisée par Ortega y Gasset et qui le conduit à distinguer deux types d'individus. Selon lui, la

30 «En vez de atender a eco sentimental de ella en nosotros, ponemos el oido y toda nuestra fijeza en los sonidos mismos, en el suceso encantador que se está realmente verificando en la orquesta. Vamos recogiendo una sonoridad tras otra, paladeándola, apreciando su color, y hasta cabria decir que su forma. », José Ortega y Gasset, « Musicalia II », op. cit. ; trad. Mathilde Pomés, José Ortega y Gasset, op. cit., p. 145-146.

31 De nouveau, nous retrouvons cette idée développée chez Schopenhauer: « Il y a dans la musique quelque chose d'ineffable et d'intime ; aussi passe-t-elle près de nous semblable à l'image d'un paradis familier quoique éternellement inaccessible ; elle est pour nous à la fois parfaitement intelligente et tout à fait inexplicable ; cela tient à ce qu'elle nous montre tous les mouvements de notre être, même les plus cachés, délivrés désormais de la réalité et de ses tourments. ", Arthur ScHOPENHAUER, Le monde comme volonté et comme représentation, op. cit., p. 337.

32 «Cuando oimos la Romanza en fa de Beethoven u otra música típicamente romántica, solemos gozar de ella concentrados hacia dentro. Vueltos, por decirlo asi, de espaldas a lo que acontece en el violín, atendemos al flujo de emociones que suscita en nosotros. No nos interesa la música por si misma, sino la repercusión mecánica en nosotros [...]. En cierto modo, pues, gozamos, no de la música, sino de nosotros mismos. [...] Yo diría que oímos la Romanza en fa, pero escuchamos el íntimo canto nuestro. », José ORTEGA Y GAsset, « Musicalia II », op. cit. ; trad. Mathilde PoMĖs, José Ortega y Gasset. Essais Espagnols, op.cit., p. 145.

33 «Es ésta sencillisima de procedimientos, pero va inspirada por una actitud espiritual radicalmente opuesta a la del vulgo. De suerte que no es impopular porque es dificil, sino que es dificil porque es impopular », José OrTega y Gasset, « Musicalia I », op. cit. ; trad. Mathilde Pomés, José Ortega y Gasset,.Essais Espagnols, op.cit., p. 130.

34 Édouard Hanslick, Du beau dans la musique. Essai de réforme de l'esthétique musicale, (trad. Charles Bannelier), Paris, Christian Bourgeois Éditeur, coll. Musique/Passé/Présent, 1986. 
musique nouvelle présuppose l'intervention de l'auditeur. Dans la capacité à fournir ou non cet effort, s'établit une distinction entre « hommes distingués » et " hommes vulgaires »" distinction sur laquelle se fonde la conception élitiste qu'Ortega y Gasset développera par la suite dans son essai:

Pendant un siècle et demi, le "peuple », la masse, a prétendu être toute la société. La musique de Stravinsky ou le drame de Pirandello ont l'efficacité sociologique de l'obliger à se reconnaitre comme ce qu'il est, comme " seulement le peuple », un simple ingrédient, parmi d'autres, de la structure sociale, une matière inerte du processus historique, un facteur secondaire du cosmos spirituel. Par ailleurs, l'art jeune contribue également à ce que les « meilleurs » se connaissent et reconnaissent dans la grisaille de la multitude et apprennent leur mission, qui consiste à être peu nombreux et à devoir combattre contre les plus nombreux ${ }^{36}$.

Dans son article "Los escritos musicales de Ortega y Gasset y su "circunstancia » histórica $»^{37}$, José M. García Laborda propose une riche contextualisation musicale à l'écriture de «Musicalia ». Cette contextualisation est principalement centrée autour de la réception de Debussy en Espagne, depuis la création du Prélude à l'Après-midi d'un faune en 1906 jusqu'aux publications sur le compositeur d'Arconada ${ }^{38}$ (1926) et de Salazar ${ }^{39}$ (1928). Comme il a déjà été souligné, cet article d'Ortega est écrit en réaction à l'accueil fort mitigé que reçoit Iberia lors de sa création par la Orquesta Filarmónica de Madrid. Le soir du 24 janvier 1921, Pérez Casas propose à son public un programme alliant musique romantique et musique nouvelle. Dans la première partie, sont interprétés Euryanthe de Weber, Año nuevo de Mariani y González puis « Le jardin enchanté de Klingsor », extrait de Parsifal. La deuxième partie est entièrement consacrée à l'exécution de La Symphonie Écossaise de Mendelssohn. Enfin, le programme se termine par la création d'Iberia de Debussy puis par la Rédemption de César Franck. Malgré le souci de Pérez Casas de réserver les nouveautés à la troisième et dernière partie du programme, rien n'y fait. Les comptes rendus qui apparaissent dans la presse sont sans équivoque: seules les deux premières parties ont reçu les faveurs du public mais au fur et à mesure de l'avancement de la soirée, les mécontentements se sont fait entendre. Ortega y Gasset évoque ainsi cet épisode au début de «Musicalia »: "Le public des concerts continue d'applaudir Mendelssohn avec frénésie et de bouder Debussy $»^{40}$. Effectivement en 1921, malgré les efforts de quelques personnalités convaincues de la nécessité de propager la musique nouvelle, la scène madrilène reste encore très largement influencée par la musique symphonique du siècle

35 José Ortega y Gasset, La deshumanización del arte, op. cit., p. 849 ; trad. Paul Aubert et Ève GiUstiniani, La déshumanisation de l'art, op. cit., p. 69.

36 "Durante siglo y medio el " pueblo", la masa, ha pretendido ser toda la sociedad. La música de Stravinsky o el drama de Pirandello tienen la eficacia sociológica de obligarle a reconocerse como lo que es, como " sólo pueblo", mero ingrediente, entre otros, de la estructura social, inerte materia del proceso histórico, factor secundario del cosmos espiritual. Por otra parte, el arte joven contribuye también a que los "mejores " se conozcan y reconozcan entre el gris de la muchedumbre y aprendan su misión, que consiste en ser pocos y tener que combatir para muchos », José ORTEGA Y GASSET, « La deshumanización del arte ", op. cit., p. 849 ; trad. Paul AUBERT et Ève GIUsTINIANI, La déshumanisation de l'art, op. cit., p. 69.

37 José M. Garcia LABORDa, «Los escritos musicales de Ortega y Gasset y su « circunstancia » histórica », Revista de Estudios Orteguianos, n ${ }^{\circ} 10-11$, mai y noviembre 2005, p. 245-272.

38 César Muñoz Arconada, En torno a Debussy, Madrid, Espasa-Calpe, 1926.

39 Adolfo Salazar, Música y músicos de hoy, Madrid, Mundo Latino, 1928.

40 «El público de los conciertos sigue aplaudiendo frenéticamente a Mendelssohn y continúa siseando a Debussy », José Ortega y Gasset, « Musicalia I », op. cit. ; trad. Mathilde Pomès, José Ortega y Gasset. Essais Espagnols, op.cit., p. 125. 
précédent. À titre d'exemple, sur les vingt-quatre opéras à l'affiche cette année-là, le Teatro Real programme pour sa soixante-et-onzième saison (1920-1921) sept œuvres de Wagner, cinq de Verdi et trois de Puccini, soit $62 \%$ des œuvres présentées.

La rédaction de l'article d'Ortegay Gasset surgit de cette comparaison entre l'enthousiasme soulevé par la musique romantique et le rejet que provoque celle de Debussy, rejet qui apparaît d'autant plus manifeste que le programme s'articulait autour de cette opposition. Dans cet article, Ortega confronte deux à deux les compositeurs emblématiques de chacun des courants auxquels il fait référence: à Beethoven et Wagner, il oppose Debussy et Stravinsky. Cette opposition schématique pose cependant quelques questions en ce qui concerne les compositeurs cités comme paradigme. La réunion de Stravinsky et de Debussy l'appellation commune de «musique nouvelle » n'est pas évidente et peut même sembler arbitraire. En 1921, Stravinsky n'est pas si impopulaire à Madrid ${ }^{41}$, ni Debussy, si moderne. En outre, aucune allusion à Falla, aux représentants de la Seconde École de Vienne ou au Groupe des Six n'apparaît dans ces écrits.

Dans l'Espagne de 1921, Stravinsky est principalement connu grâce aux deux premiers ballets qu'il a composés pour Diaghilev: l'Oiseau de feu et Petrouchka. Le public espagnol a eu l'occasion d'entendre ces œuvres - qui datent déjà d'une dizaine d'années - à chacune des saisons russes données au Teatro Real ${ }^{42}$. Lors de la cinquième saison, elles ne suscitent plus l'hostilité des premières années et sont admises au sein du répertoire du théâtre royal. Sans être populaires, elles sont appréciées pour leur dimension « folklorique ", qui apparaît comme une alternative possible à la constitution d'une musique nationale espagnole.

Depuis 1913 et la très scandaleuse création du Sacre, son compositeur a été proclamé " pape de la modernité " $^{43}$ par les élites intellectuelles et artistiques parisiennes. Avec plus de violence encore que Debussy, Stravinsky a semblé renverser l'esthétique romantique. Sept ans plus tard, le compositeur rebat pourtant totalement les cartes en se tournant vers le néoclassicisme: "Pulcinella fut une découverte du passé, l'épiphanie grâce à laquelle l'ensemble de mon œuvre à venir devint possible. C'était un regard en arrière, certes, la première histoire d'amour dans cette direction-là; mais ce fut aussi un regard dans le miroir $n^{44}$.

En Espagne, la première exécution de Pulcinella a lieu à Barcelone en 1924 et il faut attendre 1928 pour que le Sacre soit créé au Liceo. Malgré ce décalage entre l'évolution créatrice de Stravinsky et la connaissance du compositeur à Madrid, il représente la modernité musicale dans la capitale espagnole. Il faut également noter que le compositeur se trouve à Madrid lors de la publication de l'article d'Ortega puisqu'il est venu diriger Petrouchka au Teatro Real. Pendant ce séjour, il accorde un entretien dans La Voz, dans

41 On se souvient que dans « Elogio del Murciélago » écrit la même année, Ortega y Gasset cite les Ballets russes comme exemple de spectacle remportant l'adhésion systématique du public. Or en 1921, en Espagne, la musique de Stravinsky est étroitement associée à la compagnie de Diaghilev. Néanmoins, on pourra objecter que la musique du compositeur est alors un élément au sein d'un tout et que l'émotion suscitée provient de la forme du spectacle.

42 Les Ballets russes se produisent pour la première fois au Teatro Real de Madrid au mois de mai 1916. Au printemps 1917, puis à l'automne de la même année sont données les deuxième et troisième saisons russes. La quatrième saison a lieu l'année suivante. En 1921, la compagnie de Diaghilev vient se produire pour la dernière fois à Madrid.

43 Stefan JAROcinskI, «Un apôtre de l'art apollinien », Stravinsky. Études et témoignages présentés par François Lesure, Paris, J.-C. Lattès, 1982, p. 71

44 Igor Stravinsky et Robert Craft, Expositions and Developments, New York, Doubleday, 1962 ; cité et traduit par André Boucourechliev, Igor Stravinsky, Paris, Fayard, coll. Les Indispensables de la musique, 1989, p. 181. 
lequel il se présente en antiwagnérien convaincu et défend le concept de la musique pure ${ }^{45}$; propos qui ne sont évidemment pas sans faire écho à ceux que tient Ortega au même moment dans « Musicalia » et qui annoncent la « déshumanisation » de l'art à venir.

Le cas de Debussy est plus ambivalent encore tant il peut sembler surprenant que celui-ci soit cité comme figure de la modernité en 1921. Disparu trois ans plus tôt, le compositeur français commence à être combattu par l'avant-garde musicale parisienne. À la charnière entre deux siècles, le compositeur s'inspire des différentes mouvances qui lui sont contemporaines et plus particulièrement de l'impressionnisme et du symbolisme. Son appréciation oscille selon les périodes et les points de vue. Pierre Boulez, par exemple, fixait le commencement du $\mathrm{xx}^{\mathrm{e}}$ siècle en musique en 1913: Jeux de Debussy pose le point final du siècle précédent tandis que le Sacre du printemps inaugure l'avènement de la modernitét6.

Avec l'impressionnisme pictural, Debussy partage le culte panthéiste de la nature et la volonté d'évoquer l'instant dans ce qu'il peut avoir de fugace en s'adressant non plus à l'intellect, mais au ressenti du spectateur. En peinture, cette quête du naturel et de la spontanéité a conduit les artistes à renouveler leur langage et leur technique. Refusant la ligne de contour, le clair-obscur et la perspective centrale, les peintres ont évoqué l'objet par un jeu de contraste entre les couleurs. De même, en musique, Debussy tend à s'écarter des carcans de l'écriture traditionnelle en privilégiant la beauté intrinsèque des accords « en dehors de [leur] statut de consonance ou de dissonance au sein de la phrase $»^{47}$, en assouplissant les carrures et en se libérant de la forme consacrée de la symphonie. Mais les points communs s'arrêtent là. Car l'attirance du compositeur pour l'univers poétique baudelairien et verlainien, pour l'exotisme et l'ailleurs, lui fait préférer la suggestion à la description.

Mettant en musique explicitement ou implicitement ${ }^{48}$ des textes symbolistes, Debussydéfend l'idée selon laquelle l'union de la poésie et de la musique, en tant que mode intuitif de connaissance, permet d'accéder à une réalité supérieure et inaccessible à la seule raison. Ce goût pour le mystère le conduit à privilégier la discontinuité qu'il emprunte à l'écriture maeterlinckienne:
À la juxtaposition dans le syntagme de deux paradigmes sémantiques éloignés, correspond dans la mise en musique la juxtaposition dans le temps de deux éléments très différents, en particulier des échelles différentes (pentatoniques, heptatoniques, tonales, chromatiques, gammes par tons); à la dispersion dans le syntagme d'éléments appartenant à un même paradigme sémantique, correspond la dispersion dans le temps d'éléments semblables, en particuliers des thèmes et des motifs identiques ${ }^{49}$.

Cet entrelacs entre impressionnisme et symbolisme explique en partie les différentes lectures qui sont faites de l'œuvre de Debussy. Libératrice des formes pour certains, accumulation de nuances diffuses et fin de siècle pour d'autres, sa musique est loin de faire consensus au tournant des années 1920. Autour de Stravinsky gravite un front anti-

45 P. Victory, « Los grandes compositores. Una conversación con Stravinsky », La Voz, 21-III-1921, p. 1.

46 Conférence donnée par Pierre Boulez, le 30 mai 2013 à l'occasion du centenaire du Sacre du printemps, organisé par le Théâtre des Champs Élysées. « Rencontre autour du Sacre », et donnée au Théâtre de la Comédie.

47 Christian Accaour (dir.), Éléments d'esthétique musicale. Notions, formes et styles en musique, Lonrai (Normandie), Actes Sud/Cité de la musique, 2011, p. 238.

48 C'est notamment le cas du Prélude à l'Après-midi d'un Faune.

49 Christian Accaoul (dir.), Id., p. 668. 
debussyste qui compte bien «dépouiller la musique de [son] mystère ${ }^{50}$. En 1917, la création de Parade de Satie, puis la publication du Coq et l'arlequin de Cocteau l'année suivante, apparaissent comme autant de coups de force assenés au wagnérisme et au debussysme confondus:

Pelléas, c'est encore de la musique à écouter la figure dans les mains. Toute musique à écouter dans les mains est suspecte. Wagner, c'est le type de la musique qui s'écoute dans les mains.

On ne peut pas se perdre dans le brouillard Debussy comme dans la brume Wagner, mais on y attrape du $\mathrm{mal}^{51}$.

Au flou impressionniste, Cocteau oppose la simplicité et l'humour de Satie. À l'influence allemande et russe à laquelle Debussy n'a pu s'empêcher de succomber, Cocteau préfère la musique des bals populaires et des cafés-concerts français.

Face à ce front serré se positionne une autre ligne qui, notamment avec Ravel et Falla, s'enthousiasme de cette libération du langage musical et, notamment, du dépassement du développement et de la forme sonate opérés par Debussy:
Mais qui donc, considérant en toute impassibilité et conscience l'actuel renouveau de la musique européenne, pourra nier que l'œuvre de l'auteur de Pelléas en marque puissamment le point de départ?
Il ne m'échappe pas que certains des plus importants parmi les révolutionnaires actuels suivent une esthétique et même des procédés bien définis qui n'ont rien à voir avec ceux de Claude Debussy. Mais ces moyens si nouveaux, les utilisa-t-on comme aujourd'hui de manière systématique, positive et quelquefois presque exclusive, avant que Debussy, brisant les fortes chaînes qui entravaient la musique, donnât à celle-ci liberté complète, et prouvât qu'elle pouvait alors se satisfaire d'une logique, d'un équilibre et d'une perfection égale ou supérieure aux vertus de la période classique ${ }^{52}$ ?

Depuis $1915^{53}$, cette défense de la modernité musicale de Debussy est menée en Espagne par Falla et Salazar. Conférences, hommages et concerts sont organisés pour propager la nouvelle école française. En 1916, Falla publie deux articles dans la Revista musical hispano-americana dans lesquels il souligne l'importance de Debussy au sein du mouvement de rénovation de la musique européenne ${ }^{54}$. En 1918, deux hommages sont organisés à Madrid pour la mort du compositeur. Le premier a lieu à l'Ateneo sous la direction de Miguel Salvador; le second, à la Sociedad Nacional de Música, sous celle de Salazar. Les auditions des concerts qui sont alors organisés sont relayées dans El Sol qui publie de nombreux comptes rendus à ce sujet. Jusqu'en 1921, Salazar intervient très fréquemment dans les colonnes du quotidien pour analyser et diffuser les œuvres de

50 Igor Stravinsky, cité par Jean-Dominique Krynen, in Federico Sopeña, Manuel de Falla, Écrits sur la musique et sur les musiciens, trad. Jean-Dominique Krynen, Arles, Actes Sud Beaux Arts, 1992, p. 46 , p. 104.

51 Jean Cocteau, Le coq et l'arlequin, Clamecy, Éditions Stock, 2009 [1918], p. 79.

52 Manuel de FALLA, « Prologue de La musique française contemporaine », paru dans La Revista musical hispano-americana, ${ }^{\circ} 8$, juillet 1916, p. 2 ; article reproduit in Federico Sopeña, Manuel de Falla. Écrits sur la musique et sur les musiciens, op. cit., p. 59.

53 C'est en 1915, à l'Ateneo de Madrid, que Manuel de Falla prononce la conférence « Introducción a la música nueva » dans laquelle le compositeur traite de l'importance de Debussy et de l'impressionnisme.

54 Manuel DE FALLA, "Prólogo de la Música francesa contemporánea », Revista musical hispanoamericana, Madrid, juillet 1916, p. 2 - 4 ; «Introducción al estudio de la música nueva », Revista musical hispano-americana, Madrid, 31 décembre 1916, p. 2 - 5 ; ce second article reprend les propos en faveur de la musique nouvelle que le compositeur avaient tenus lors de la conférence donnée l'année précédente à l'Ateneo de Madrid. 
Debussy. En 1919, par exemple, il consacre un article à la création au Tivoli de Barcelone de Pelléas et Mélisande ${ }^{55}$.

Cette présentation de Debussy comme compositeur emblématique de la modernité ${ }^{56}$ ne relève pas d'une méconnaissance de la part d'Ortega y Gasset et encore moins d'un éventuel «retard» de l'Espagne ${ }^{57}$. Le philosophe a lu l'essai de Cocteau paru en $1918^{58}$ et assiste aux conférences et concerts organisés par l'Ateneo, le Círculo de Bellas Artes et la Residencia de Estudiantes. Sa position s'aligne en réalité sur celle des défenseurs de la modernité que sont Falla et Salazar ${ }^{59}$. Bien que Debussy et Stravinsky proposent des esthétiques distinctes, ce qui importe pour Ortega ce sont les possibilités de rénovation qu'ils offrent. Peu importe si leur réunion sous le terme de « musique nouvelle » semble arbitraire, tous deux, et pour différentes raisons, incarnent des aspects de la modernité musicale. Si le philosophe se refuse à utiliser le terme « d'impressionnisme » pour évoquer Debussy, c'est bien parce qu'il renvoie à une esthétique qui appartient au siècle précédent et qui apparaît comme un dernier avatar du réalisme.

La référence à Debussy relève donc d'un choix délibéré. Cocteau reviendra d'ailleurs rapidement sur ses déclarations et justifiera le ton péremptoire employé en avançant l'argument de son jeune âge ${ }^{60}$. Quant à Falla, son admiration inconditionnelle tient principalement à deux raisons. D'une part, Debussy est le premier à s'être libéré de l'hégémonie allemande, bien plus que Strauss ou Schönberg selon lui. D'autre part, il est également le premier à réussir le tour de force d'évoquer l'Espagne sans avoir recours aux clichés rebattus.

Ortega y Gasset, pour sa part, ne consacrera plus aucun écrit à la musique. Mais il se souviendra de cette analyse au moment de réfléchir sur les différents mouvements de l'avant-garde. Il énoncera qu'à l'instar du champ musical, la modernité dans l'art se caractérise par un même refus du mimétisme, une préoccupation essentiellement formelle et une jouissance avant tout esthétique.

55 Adolfo Salazar, « En el Tivoli. Pelléas et Melisande », El Sol, 20 octobre 1919, p. 5.

56 Les Espagnols ne sont pas les seuls à considérer Debussy comme le compositeur initiateur de la modernité musicale. À titre d'exemple, Bela Bartók le cite fréquemment comme le plus grand compositeur du xx $x^{e}$ siècle et en 1939, disait au chef d'orchestre Serge Moreux: « Debussy a restauré le sens des accords chez tous les musiciens ; il a été aussi important que Beethoven qui, lui, nous a révélé la forme progressive, et que Bach qui nous a définitivement introduits à la transcendance du contrepoint. », Jean et Brigitte MAssin (dir.), Histoire de la musique occidentale, Paris, Fayard, coll. Les Indispensables de la musique, 1993, p. 1004.

57 Falla, lors de sa conférence sur la musique nouvelle de 1915, inscrit les recherches de Schönberg au sein d'un mouvement plus ample, qui s'initie avec Debussy.

58 Le philosophe cite très brièvement Cocteau dans « Musicalia ».

59 Lors de cette même conférence de 1915, Falla cite comme compositeurs emblématiques du siècle précédent Beethoven et Wagner, choix qui sera repris par le philosophe dans ses écrits. Le choix des quatre compositeurs cités par Ortega y Gasset souligne la connaissance ainsi que la proximité de sa pensée avec celle de Manuel de Falla.

60 Jean Cocteau ne maintiendra pas longtemps sa position anti-debussyste. Dès la fin des années 1920 , il commence à sentir le besoin de revenir sur les propos tenus dans le coq et l'arlequin. En 1930, il justifie ses déclarations par la nécessité de défendre la nouvelle musique française des Six contre l'impressionnisme. Dans les années 1940, il affirmera son admiration pour Debussy et son chef-d'œuvre Pelléas et Mélisande. En 1962, il en concevra les décors et les costumes pour une production qui aura lieu à Metz. 